
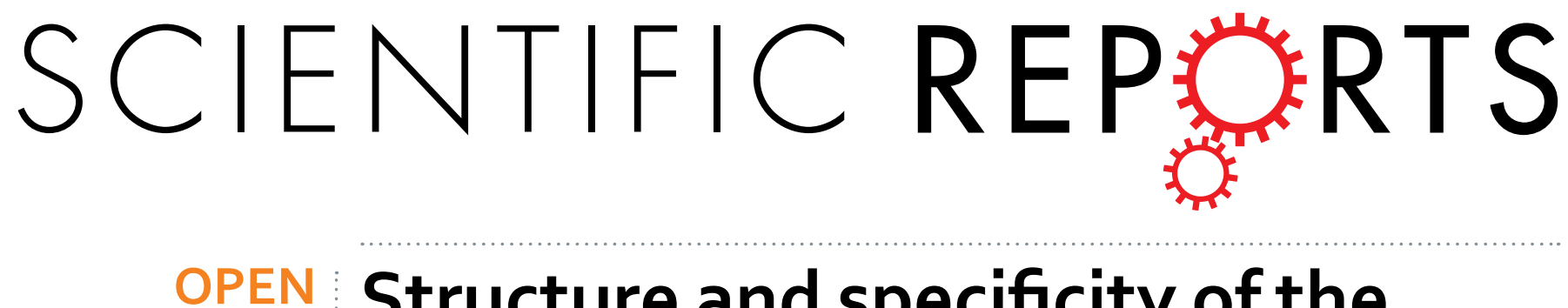

\title{
Structure and specificity of the
} Type VI secretion system ClpV-TssC interaction in enteroaggregative

Received: 06 June 2016

Accepted: 13 September 2016

Published: 04 October 2016

\section{Escherichia coli}

Badreddine Douzi ${ }^{1,2}{ }^{2}$, Yannick R. Brunet ${ }^{3,}{ }^{+}$, Silvia Spinelli ${ }^{1,2}$, Valentine Lensi $^{3}$, Pierre Legrand ${ }^{4}$, Stéphanie Blangy ${ }^{1,2}$, Anant Kumar ${ }^{3}$, Laure Journet ${ }^{3}$, Eric Cascales ${ }^{3}$ \& Christian Cambillau ${ }^{1,2}$

The Type VI secretion system (T6SS) is a versatile machine that delivers toxins into either eukaryotic or bacterial cells. It thus represents a key player in bacterial pathogenesis and inter-bacterial competition. Schematically, the T6SS can be viewed as a contractile tail structure anchored to the cell envelope. The contraction of the tail sheath propels the inner tube loaded with effectors towards the target cell. The components of the contracted tail sheath are then recycled by the ClpV AAA ${ }^{+}$ATPase for a new cycle of tail elongation. The T6SS is widespread in Gram-negative bacteria and most of their genomes carry several copies of T6SS gene clusters, which might be activated in different conditions. Here, we show that the ClpV ATPases encoded within the two T6SS gene clusters of enteroaggregative Escherichia coli are not interchangeable and specifically participate to the activity of their cognate T6SS. Here we show that this specificity is dictated by interaction between the $\mathrm{ClpV} \mathrm{N}$-terminal domains and the $\mathrm{N}$-terminal helices of their cognate Tss $\mathrm{C} 1$ proteins. We also present the crystal structure of the ClpV1 N-terminal domain, alone or in complex with the Tss $\mathrm{C} 1 \mathrm{~N}$-terminal peptide, highlighting the commonalities and diversities in the recruitment of $\mathrm{ClpV}$ to contracted sheaths.

The Type VI secretion system (T6SS) is a multi-protein complex widely distributed in Gram-negative bacteria with an over-representation in Proteobacteria and Bacteriodetes responsible for the transport and delivery of effector toxins into target cells ${ }^{1-4}$. The activities and molecular targets of the T6SS effectors correlate with the specific needs of the bacterium in its environmental niche. In most bacteria, the T6SS confers a competitive advantage in multi-species environments, as it delivers anti-bacterial toxins with peptidoglycan hydrolase, phospholipase or DNase activity into target bacterial cell $s^{5-8}$. The T6SS thus regulates bacterial populations and facilitates colonization of the environment ${ }^{9}$. In addition to its role in the bacterial warfare, a few T6SS have been shown to secrete toxins that are active in eukaryotic cells, such as proteins that interfere with the actin or tubulin assembly pathways ${ }^{10-13}$. The T6SS comprises 13 conserved and essential components named TssA to TssM ${ }^{14,15}$. These core-components assemble two sub-complexes ${ }^{15-17}$. The first sub-complex is evolutionarily, structurally and functionally similar to the tail structures of contractile bacteriophages ${ }^{14,18,19}$. It is constituted of a $\sim 600 \mathrm{~nm}$-long inner tube made of Hcp hexamers stacked on each other, and wrapped into a sheath-like structure ${ }^{20,21}$. The sheath-like structure is composed of rows of heterodimers of TssB and TssC (VipA and VipB in Vibrio cholerae), which assemble into an extended, high-energy conformation ${ }^{16,18,20,22-24}$. The Hcp inner tube is tipped by the VgrG/PAAR complex, that serves as puncturing device for the penetration of the target cell, as well as adaptor complex for effectors ${ }^{25-29}$. The VgrG spike

${ }^{1}$ Architecture et Fonction des Macromolécules Biologiques (AFMB, UMR 6098), Centre National de la Recherche Scientifique (CNRS), Campus de Luminy, Case 932, 13288 Marseille Cedex 09, France. ${ }^{2}$ Architecture et Fonction des Macromolécules Biologiques (AFMB, UMR 6098), Aix-Marseille Univ, Campus de Luminy, Case 932, 13288 Marseille Cedex 09, France. ${ }^{3}$ Laboratoire d'Ingénierie des Systèmes Macromoléculaires (LISM, UMR 7255), Institut de Microbiologie de la Méditerranée (IMM), Centre National de la Recherche Scientifique (CNRS), Aix-Marseille Univ, 31 chemin Joseph Aiguier, 13402 Marseille Cedex 20, France. ${ }^{4}$ Synchrotron Soleil, L'Orme des Merisiers, Saint-Aubin - BP 48, 91192 Gif-sur-Yvette Cedex, France. 'Present address: Department of Microbiology and Immunobiology, Harvard Medical School, 77 Avenue Louis Pasteur, Boston, MA, 02115 USA. Correspondence and requests for materials should be addressed to E.C. (email: cascales@imm.cnrs.fr) or C.C. (email: cambillau@afmb.univ-mrs.fr) 
protein is also part of the assembly platform composed of the TssEFGK proteins ${ }^{30,31}$. This platform - or baseplate - controls the polymerization of the tube/sheath structure and probably initiates sheath contraction ${ }^{31,32}$. This platform is tightly attached to the cell envelope through multiple contacts with components of the second sub-complex, called membrane complex ${ }^{31,33}$. The membrane complex is composed of three proteins: the inner membrane TssL and TssM proteins and the TssJ outer membrane lipoprotein ${ }^{34-38}$. These three proteins are present in 10 copies each and assemble a 1.7-MDa complex that crosses the cell envelope and delimits a channel for the passage of the inner tube during sheath contraction ${ }^{39}$. Once in contact with a target cell, or when the cell senses an attack by a competitor, the T6SS assembles the tubular structure and the sheath contracts, hence propelling the inner tube/spike towards the target cell and delivering toxins $s^{20,40}$. While the membrane complex is stable and can be reused for multiple injections ${ }^{39}$, the contracted sheath is disassembled by a dedicated AAA $\mathrm{ATPa}^{+} \mathrm{ATP}_{\text {of the Clp }}$ family, ClpV, and recycled for a new assembly and injection cycles ${ }^{22,23,41}$. In most T6SS, ClpV is recruited to the contracted sheath via interactions with the $\mathrm{N}$-terminal helix of the TssC subunits that is thought to be accessible only in the contracted conformation $22,23,41-43$. The $\mathrm{N}$-terminal helix of Tss $\mathrm{C}$ accommodates into a cleft constituted of charged residues located at the interface of two helices, called $\mathrm{H} 1$ and $\mathrm{H} 2$, at the $\mathrm{N}$-terminus of $\mathrm{ClpV}^{41,43}$. However, in a subset of T6SS, the cleft is composed of uncharged residues, and the ClpV-TssC interaction is mediated or stabilized by the TagJ accessory protein ${ }^{43}$.

Interestingly, most bacteria encode several copies of T6SS gene clusters in their genomes ${ }^{1-3,44}$. These clusters are usually responsive to different environmental cues and hence subjected to different regulatory mechanisms ${ }^{45}$. It is thought that this diversity is responsible for the activation of distinct T6SS machineries in different conditions. However, little is known on how the subunits select their cognate machineries when multiple paralogous T6SS are present in the same cell. Enteroaggregative Escherichia coli strain 17-2 encodes two T6SS gene clusters of the T6SS-1 and T6SS-3 sub-families ${ }^{44}$, and it has been shown that the inner tube component Hcp encoded by the T6SS-1 cluster (sci-1) specifically interacts with the sheath component TssB1, but not with TssB2, which is encoded by the T6SS- 3 cluster (sci-2), demonstrating specificity during assembly of the T6SS tails ${ }^{21}$. Here, we report that specificity also applies to disassembly of the contracted sheath. We demonstrate that over-production of ClpV2 cannot compensate for the absence of ClpV1 and cannot restore functionality of the T6SS-1 (and vice versa). We further provide evidence that $\mathrm{ClpV} 1$ specifically interacts with the $\mathrm{N}$-terminal helix of TssC1 and not with that of TssC2. We then report the crystal structure of the ClpV1 N-terminal domain alone and in complex with the TssC1 N-terminal helix. Noteworthy, the crystal structure of the complex differs significantly from what was reported for ClpV-VipB complex from $V$. cholerae ${ }^{41}$. Our results suggest an alternative mode of binding between TssC1 and ClpV1, as the cleft in ClpV1 is essentially composed of uncharged residues but does not require TagJ to efficiently bind to the TssC1 N-terminal helix.

\section{Experimental Procedures}

Bacterial strains and media. The Escherichia coli K-12 DH5 $\alpha$, BTH101, W3110 and BL21(DE3) pLysS strains were used for cloning procedures, bacterial two-hybrid analyses, co-immunoprecipitations and protein production, respectively. Strain W3110 pUA66- $r r n B\left(\mathrm{Kan}^{\mathrm{R}}, \mathrm{GFP}^{+}\right)^{46}$ was used as prey in anti-bacterial competition experiments. Cells were grown in Lysogeny broth (LB), Sci-1-inducing medium (SIM) or Dulbelcco modified Eagle medium (DMEM), as specified. Plasmids were maintained by the addition of ampicillin $(100 \mu \mathrm{g} / \mathrm{mL})$, chloramphenicol $(40 \mu \mathrm{g} / \mathrm{mL})$ or kanamycin $(50 \mu \mathrm{g} / \mathrm{mL})$.

Plasmid construction for in vivo studies. Plasmids used in this study are listed in Supplemental Table S1. Polymerase Chain Reactions (PCR) were performed using a Biometra thermocycler using the Q5 high fidelity DNA polymerase (New England BioLabs). Custom oligonucleotides, listed in Supplemental Table S1, were synthesized by Sigma Aldrich. Enteroaggregative E. coli 17-2 chromosomal DNA was used as a template for all PRCs. The amplified DNA fragments correspond to the full-length ClpV1 (EC042_4530, GI: 284924251), ClpV2 (EC042_4577, GI: 284924293), TssC1 (EC042_4525, GI: 284924246) and TssC2 (EC042_4562, GI: 284924279) proteins, as well as the N-terminal domains of ClpV1 (residues 1-163) and ClpV2 (residues 1-147). Plasmids were engineered by restriction-free cloning ${ }^{47}$ as previously described ${ }^{35}$. Briefly, genes of interest were amplified with oligonucleotides introducing extensions annealing to the target vector. The double-stranded product of the first PCR was then been used as oligonucleotides for a second PCR using the target vector as template. Deletion of TssC1 and TssC2 N-terminal helices as well as point mutations have been obtained by site-directed mutagenesis. All constructs have been verified by restriction analysis and DNA sequencing (Eurofins, MWG).

Bacterial two-hybrid assay. The adenylate cyclase-based bacterial two-hybrid technique ${ }^{48}$ was used as previously published ${ }^{49}$. Briefly, compatible vectors producing proteins fused to the isolated T18 and T25 catalytic domains of the Bordetella adenylate cyclase were transformed into the reporter BTH101 strain and the plates were incubated at $30^{\circ} \mathrm{C}$ for 24 hours. Three independent colonies for each transformation were inoculated into $600 \mu \mathrm{L}$ of LB medium supplemented with ampicillin, kanamycin and IPTG $(0.5 \mathrm{mM})$. After overnight growth at $30^{\circ} \mathrm{C}, 10 \mu \mathrm{L}$ of each culture were spotted onto LB plates supplemented with ampicillin $(100 \mu \mathrm{g} / \mathrm{mL})$, kanamycin $(50 \mu \mathrm{g} / \mathrm{mL})$, IPTG $(0.5 \mathrm{mM})$ and Bromo-Chloro-Indolyl-Galactopyrannoside (X-Gal, $40 \mu \mathrm{g} / \mathrm{mL}$ ) and incubated for 16 hours at $30^{\circ} \mathrm{C}$. The experiments were done at least in triplicate and a representative result is shown.

Co-immunoprecipitations. $100 \mathrm{~mL}$ of W3110 cells producing the proteins of interest were grown to an absorbance at $\lambda=600 \mathrm{~nm}\left(A_{600}\right) \sim 0.4$ and the expression of the cloned genes were induced with AHT $(0.1 \mu \mathrm{g} / \mathrm{mL})$ and L-arabinose $(0.2 \%)$ for $45 \mathrm{~min}$. The cells were harvested, and the pellets were resuspended in $20 \mathrm{mM}$ Tris- $\mathrm{HCl}$ pH 8.0, $100 \mathrm{mM} \mathrm{NaCl}, 30 \%$ sucrose, $1 \mathrm{mM}$ EDTA, lysozyme $100 \mu \mathrm{g} / \mathrm{mL}$, DNase $100 \mu \mathrm{g} / \mathrm{mL}$, RNase $100 \mu \mathrm{g} / \mathrm{mL}$ supplemented with protease inhibitors (Complete, Roche) to an $A_{600}$ of 80 and incubated on ice for 20 min. Cells were lysed by three passages at the French Press ( 800 psi) and lysates were clarified by centrifugation at 
$20,000 \times g$ for $20 \mathrm{~min}$. Supernatants were used for co-immunoprecipitation using anti-FLAG M2 affinity gel (Sigma-Aldrich). After 3 hours of incubation, the beads were washed three times with $1 \mathrm{~mL}$ of $20 \mathrm{mM} \mathrm{Tris-HCl}$ $\mathrm{pH} 8.0,100 \mathrm{mM} \mathrm{NaCl}, 15 \%$ sucrose, resuspended in $25 \mu \mathrm{L}$ of Laemmli loading buffer, boiled for $10 \mathrm{~min}$ and subjected to SDS-PAGE and immunodetection analyses.

Anti-bacterial competition assay. Antibacterial competition growth assays were performed as previously described, in Sci-2-inducing ${ }^{40}$ or Sci-1-inducing ${ }^{29}$ conditions. The wild-type E. coli strain W3110 bearing the kanamycin-resistant $\mathrm{GFP}^{+}$pUA66-rrnB plasmid $^{46}$ was used as prey.

Plasmid construction for in vitro studies. The DNA sequence encoding the ClpV1 N-terminal domain (ClpV1-Nt, Gly1 to Leu163) was cloned into the pETG-20A expression vector using standard Gateway procedures to yield pETG20A-ClpV1-Nt. The resulting construction allows the production of ClpV1-Nt fused to an $\mathrm{N}$-terminal Thioredoxin followed by a $6 \times$ His tag and a Tobacco Etch Virus (TEV) cleavage site. Primers used for genes amplification are shown in Supplemental Table S1.

Peptide synthesis, protein production and characterization. E. coli BL21 (DE3) pLysS cells carrying the pETG20A-ClpV1-Nt plasmid were grown at $37^{\circ} \mathrm{C}$ in TB medium $(1.2 \%$ peptone, $2.4 \%$ yeast extract, $72 \mathrm{mM}$ $\mathrm{K}_{2} \mathrm{HPO}_{4}, 17 \mathrm{mM} \mathrm{KH}_{2} \mathrm{PO}_{4}$, and $0.4 \%$ glycerol). Expression of $c l p V 1-N t$ was induced at $A_{600}=0.6$ with $0.5 \mathrm{mM}$ IPTG for 18 hours at $25^{\circ} \mathrm{C}$. Cells were then resuspended in lysis buffer $(50 \mathrm{mM}$ Tris- $\mathrm{HCl} \mathrm{pH} \mathrm{8.0,300} \mathrm{mM} \mathrm{NaCl}$, $1 \mathrm{mM}$ EDTA, $0.5 \mathrm{mg} / \mathrm{mL}$ lysozyme, $1 \mathrm{mM}$ phenylmethylsulfonyl fluoride), submitted to several freeze-thawing cycles and sonicated after the addition of $20 \mu \mathrm{g} / \mathrm{mL}$ DNase and $20 \mathrm{mM} \mathrm{MgCl}_{2}$. Pellet and soluble fraction were separated by centrifugation for $30 \mathrm{~min}$ at $16,000 \times g$. The soluble fraction containing ClpV1-Nt was loaded onto a 5-mL Ni ${ }^{2+}$ affinity column (HisTrap ${ }^{\mathrm{TM}} \mathrm{FF}$ ) using an ÄKTA Explorer apparatus (GE healthcare) and the immobilized proteins were eluted in $50 \mathrm{mM}$ Tris- $\mathrm{HCl} \mathrm{pH} 8.0,300 \mathrm{mM} \mathrm{NaCl}$ supplemented with $250 \mathrm{mM}$ imidazole. The protein solution was desalted on a HiPrep 26/10 column (Sephadex ${ }^{\mathrm{TM}}$ G-25, Amersham Biosciences), and ClpV1-Nt was obtained by cleavage using $2 \mathrm{mg}$ of TEV protease for 18 hours at $4{ }^{\circ} \mathrm{C}$ and collected in the flow-through of a 5-mL Nickel column. The protein was concentrated using the centricon technology (Millipore, $10-\mathrm{kDa}$ cut-off). After concentration, the soluble ClpV1-Nt protein was passed through a Sephadex 200 26/60 column pre-equilibrated with $25 \mathrm{mM}$ Tris- $\mathrm{HCl}$ pH7.5, $100 \mathrm{mM} \mathrm{NaCl}, 5 \%$ Glycerol. A similar procedure was applied for the ClpV2-Nt domain but the protein was insoluble and could not be purified.

Peptides corresponding to the $\alpha$-helices from TssC1 and TssC2 were synthesized by Genscript (TssC1 (residues 23-35), KKW-DSVYASLFEKINL-KK; TssC2 (residues 15-29), ATDDCLEEIINNTRA). Due to its hydrophobic nature, the TssC1 N-terminal peptide was flanked by two lysines to improve its solubility and by an additional tryptophane to follow it during purification.

Microscale thermophoresis (MST) experiments. Microscale thermophoresis experiments were performed using a Monolith NT.115 apparatus (NanoTemper). ClpV1-Nt was labeled with the blue-fluorescent dye NT-495-NHS (NanoTemper) and the buffer was exchanged for the assay buffer (25 mM Tris-HCl pH 7.5, $200 \mathrm{mM} \mathrm{NaCl}$, glycerol 5\%, 0.05\% Tween-20) using a Nap5 column (GE Healthcare). Titrations were conducted with a constant $200 \mathrm{nM}$ fluorophore-labeled ClpV1-Nt against up to $500 \mu \mathrm{M}$ TssC N-terminal peptides in hydrophilic-coated capillaries. Each data point was measured in triplicate. Single-site fitting was performed using the NanoTemper data analysis software.

Crystal structures determination. ClpV1-Nt was purified to homogeneity and the protein crystallized spontaneously during gel filtration. ClpV1-Nt crystals belong to space group $\mathrm{P} 2{ }_{1} 2_{1} 2_{1}$ with cell dimensions $\mathrm{a}=40.9 \AA, \mathrm{b}=58.7 \AA$, $\mathrm{c}=65.6 \AA$ (Supplemental Table S2). A complete data set was collected at the Soleil synchrotron (Saint-Aubin, France) at $2 \AA$. Data were integrated with $\mathrm{XDS}^{50}$ and reduced with XSXALE ${ }^{50}$ (Supplemental Table S2). The structure was solved by soaking crystals in a $0.5 \mathrm{M} \mathrm{NaI}$ and $0.5 \mathrm{M}$ of CsI solution followed by data collection at 1.77- $\AA$ wavelength, allowing remote SAD phasing. The cesium/iodine positions were defined with SHELXC and SHELXD ${ }^{51}$ and subsequently used for SAD phasing using PHASER ${ }^{52}$. These phases were improved by solvent flattening and histogram matching using the program PARROT ${ }^{53}$, resulting in interpretable maps from which the BUCCANEER program ${ }^{54}$ was able to build an initial model. The model was completed and corrected manually with COOT $^{55}$ and refined with autoBUSTER ${ }^{56}$ (Supplemental Table S2).

The ClpV1-Nt/TssC1 peptide complex was obtained by mixing the purified ClpV1 N-terminal domain with the peptide at a 1:4 molar ratio in $20 \mathrm{mM}$ Tris- $\mathrm{HCl} \mathrm{pH} 8.0,100 \mathrm{mM} \mathrm{NaCl}, 5 \%$ Glycerol and concentrated using the centricon technology (Millipore, $\mathrm{kDa}$ cut-off of 10) to $2 \mathrm{mg} / \mathrm{mL}$. Crystals were obtained in one condition $(0.2 \mathrm{M}$ imidazole, malate $\mathrm{pH}$ 6.0, 8\% w/v PEG 4000) using the STURA screen (Molecular dimensions) and diffracted at $2.5-\AA$ r resolution. The structure was solved by molecular replacement using the structure of ClpV1-Nt as starting model. The model was completed and corrected manually with COOT $^{55}$ and refined with autoBUSTER ${ }^{56}$ (Supplemental Table S2).

Data deposition. The EAEC ClpV1-Nt and ClpV1-Nt/TssC1 peptide X-ray structures have been deposited in the Protein Data Bank (PDB) under accession numbers $4 \mathrm{HH} 5$ and $4 \mathrm{HH} 6$, respectively.

\section{Results}

ClpV1 and ClpV2 are not interchangeable. The enteroaggregative E. coli Sci-1 and Sci-2 T6SS are both involved in bacterial competition ${ }^{29,40}$. However, these two T6SS are active in different laboratory conditions 
A
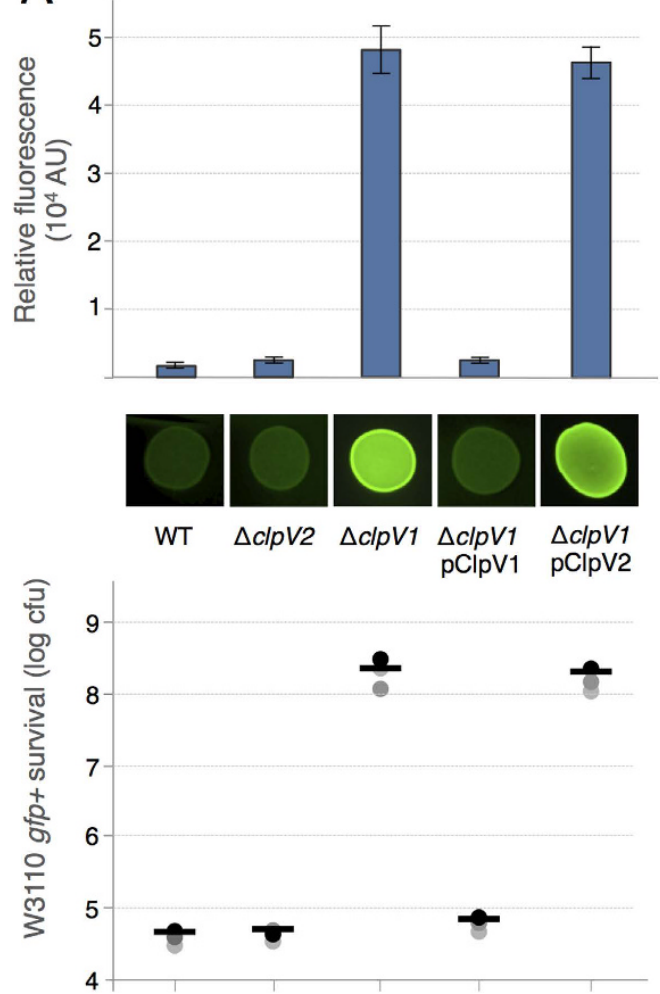

B
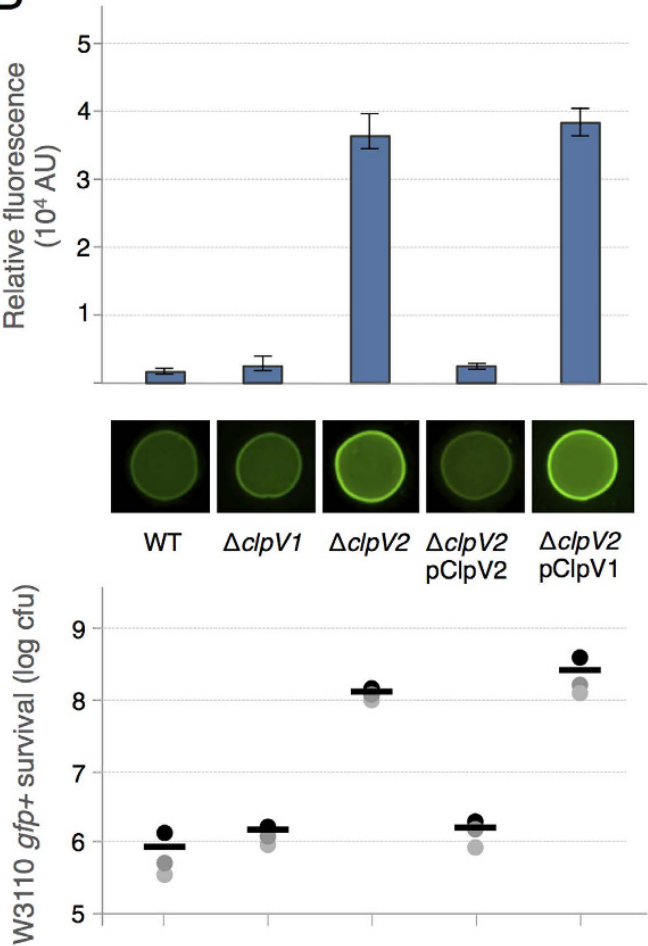

Figure 1. ClpV1-ClpV2 interchangeability. (A) Sci-1-dependent antibacterial growth inhibition. Prey cells $\left(\mathrm{W} 3110 \mathrm{gfp} \mathrm{p}^{+}, \mathrm{kan}^{\mathrm{R}}\right.$ ) were mixed with the indicated attacker cells, spotted onto sci-1-inducing medium (SIM) agar plates and incubated for 4 hours at $37^{\circ} \mathrm{C}$. (B) Sci-2-dependent antibacterial growth inhibition. Prey cells $\left(\mathrm{W} 3110 \mathrm{gfp} \mathrm{p}^{+}, \mathrm{kan}^{\mathrm{R}}\right.$ ) were mixed with the indicated attacker cells, spotted onto Dulbecco's modified Eagle Medium (DMEM) agar plates and incubated for 4 hours at $37^{\circ} \mathrm{C}$. The image of a representative bacterial spot is shown and the relative fluorescent levels (in arbitrary units, AU) are indicated in the upper graph. The number of recovered E. coli prey cells is indicated in the lower graph (in $\log _{10}$ of colony-forming units (cfu)). The circles indicate values from three independent assays, and the average is indicated by the bar.

(minimal medium for the Sci-1 T6SS and modified Eagle medium for the Sci-2 T6SS ${ }^{57,58}$ ). To test whether the two ClpV proteins, ClpV1 (EC042_4530, GI: 284924251) and ClpV2 (EC042_4577, GI: 284924293), respectively encoded by the $s c i-1$ and $s c i-2$ gene clusters are interchangeable, we engineered $\Delta c l p V 1$ and $\Delta c l p V 2$ strains and complementation vectors (pIBA-ClpV1 and pIBA-ClpV2). The different combinations were tested for their ability to confer a growth advantage in Sci-1- or Sci-2-active conditions against E. coli K-12. Figure 1 shows that while $c l p V 1$ and $c l p V 2$ trans-expression restores the growth advantage of the $\Delta c l p V 1$ and $\Delta c l p V 2$ mutant strains respectively, over-production of $\mathrm{ClpV} 2$ does not restore $\Delta c l p V 1$ defects, and vice versa. From this experiment, we concluded that the ClpV1 and ClpV2 ATPase are not interchangeable and we hypothesized that this specificity could be imputable to specific contacts with partners.

ClpV specificity is dictated by ClpV-TssC complex formation. To identify ClpV1 partners, we performed a systematic bacterial two-hybrid analysis. ClpV1 was fused to the $\mathrm{N}$ - or C-terminus of the Bordetella pertussis Cya T25 domain and these protein fusions were tested as baits against preys comprising fusion of the T18 domain to the individual T6SS core components. Growth on reporter plates demonstrated that $\mathrm{ClpV1}$ interacts with itself (Fig. 2A), in agreement with the ability of $\mathrm{AAA}^{+}$ATPases to assemble hexameric structures $^{22,59}$. However, ClpV1 does not interact with ClpV2 suggesting that these two proteins cannot assemble hetero-hexamers (Fig. 2B). In addition, ClpV1 interacts with TssC1 (EC042_4525, GI: 284924246), one of the sheath components (Fig. 2C). The ClpV1-TssC1 interaction was further confirmed by co-immunoprecipitation (Fig. 2D). The ClpV-TssC interaction has already been reported in $V$. cholerae and $P$. aeruginosa and involves contacts between the $\mathrm{N}$-terminal domain of $\mathrm{ClpV}$ and an $\mathrm{N}$-terminal helix of TssC $\mathrm{C}^{22,41,43}$. Similarly, co-immunoprecipitation assays showed that the N-terminal domain of ClpV1 (ClpV1-Nt, residues 1-163) is sufficient to mediate the interaction with TssC1, while a deletion of the TssC1 N-terminal helix (deletion of residues 23-25, TssC1 $\Delta \mathrm{h}$ ) abolishes ClpV1-TssC1 complex formation (Fig. 2D, left panel). Identical results were obtained for ClpV2 and the Sci-2-encoded TssC2 protein (Fig. 2D, right panel). We then tested cross-interaction between the ClpV N-terminal domains and TssC proteins. Fig. 2D (right lanes in left and right panels) shows that each ClpV N-terminal domain interacts only with the TssC encoded within the same gene cluster. The lack of cross-interaction between the ClpV and TssC proteins from the two T6SSs was further confirmed by bacterial two-hybrid (Fig. 2E). From these results, we concluded that specificity determinants control ClpV-TssC complex 
A

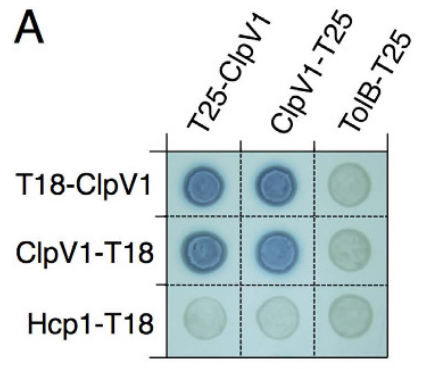

B

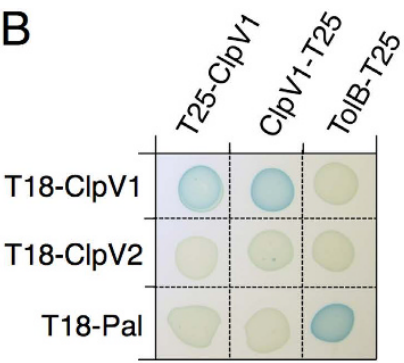

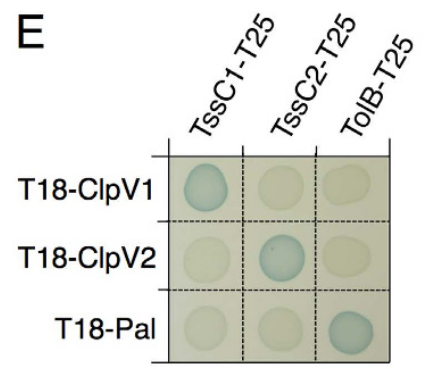

C

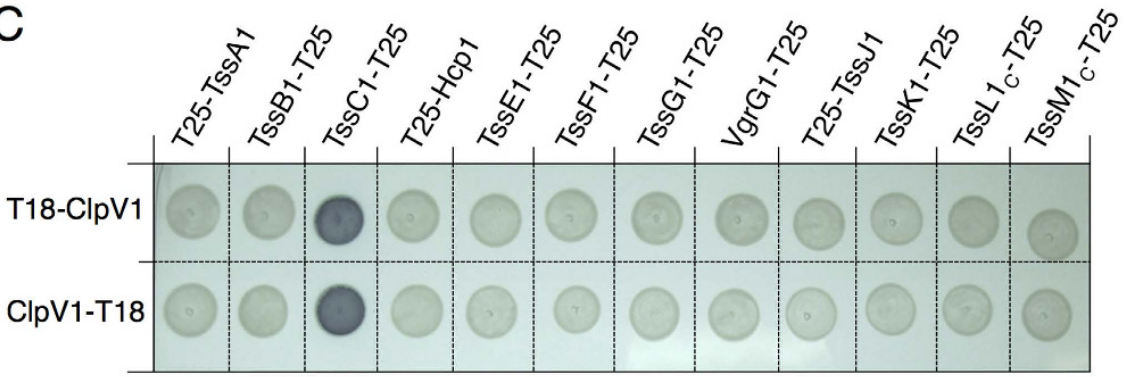

$\mathrm{D}$

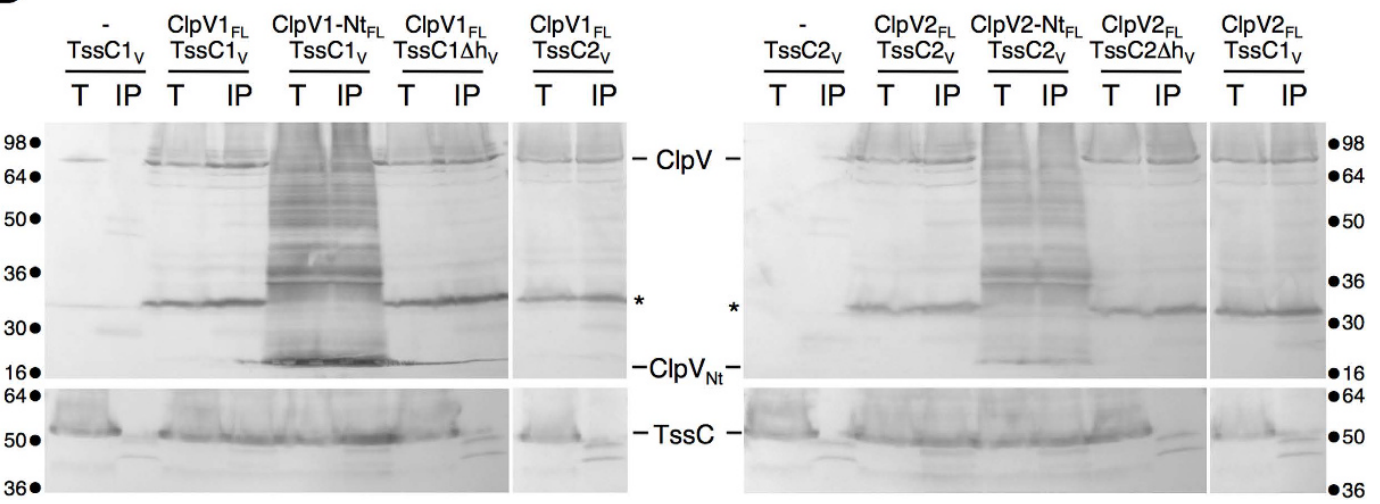

Figure 2. Interaction of the ClpV N-terminal domain with its cognate TssC protein. (A-C,E) Bacterial two-hybrid assay. BTH101 reporter cells producing the indicated proteins or domains fused to the T18 or T25 domain of the Bordetella adenylate cyclase were spotted on X-Gal indicator plates. The blue color of the colony reflects the interaction between the two proteins. (D) Co-immunoprecipitation assay. The soluble lysate from $10^{11}$ E. coli K-12 W3110 cells producing the indicated proteins ( ${ }_{\mathrm{FL}}$, FLAG-tagged; ${ }_{\mathrm{V}}$, VSVG-tagged) were subjected to immune precipitation on anti-FLAG-coupled agarose beads. The immunoprecipitated material was subjected to $12.5 \%$-acrylamide SDS-PAGE and immunodetected with anti-FLAG (upper panel) and anti-VSVG (lower panel) monoclonal antibodies. Molecular weight markers (in $\mathrm{kDa}$ ) are indicated.

formation and we hypothesized that these specificity determinants should be located within the TssC $\mathrm{N}$-terminal helices. In support of this hypothesis, a sequence alignment of $\mathrm{Tss} \mathrm{C} 1$ and $\mathrm{Tss} \mathrm{C} 2$ showed that the two proteins share high level of homologies except in the $\mathrm{N}$-terminal region that is, in average, less conserved (18\% identity within the $55 \mathrm{~N}$-terminal residues compared to $38 \%$ identity on full-length sequences, Supplemental Figure S1).

To gain further insights onto the binding and specificity of $\mathrm{ClpV} 1$ towards the Tss $\mathrm{C} \mathrm{N}$-terminal helices, the ClpV1 N-terminal domain was purified. In addition, peptides corresponding to TssC1 (residues 23-35) and TssC2 (residues 15-29) N-terminal helices (Fig. 3A) were synthesized. Binding of the TssC peptides to ClpV1-Nt was assayed by microscale thermophoresis that allows the quantification of molecular interactions in solution. Figure $3 \mathrm{~B}$ shows that the TssC1 peptide binds to ClpV1-Nt with a $\mathrm{K}_{D}$ of $\sim 26 \mu \mathrm{M}$, a value that is comparable to the affinity measured between $V$. cholerae $\mathrm{ClpV}$ and the $\mathrm{VipB}$ (TssC) N-terminal peptide $\left(\mathrm{K}_{D} \sim 39 \mu \mathrm{M}\right)^{41}$. By contrast, no binding of TssC2 peptide to ClpV1-Nt was observed (Fig. 3C).

Structures of the ClpV1 N-terminal domain alone and in complex with the Tss $\mathrm{C} 1 \mathrm{~N}$-terminal peptide reveal a new mode of binding. To obtain molecular details on the ClpV1-TssC1 interaction, we crystallized the ClpV1-Nt domain alone and in complex with the TssC1 N-terminal helix peptide. ClpV1-Nt crystallized in the $\mathrm{P} 2{ }_{1} 2_{1} 2_{1}$ space group and diffracted to 2.0 - $\AA$ resolution (Supplemental Table S2). ClpV1-Nt structure was solved using a CsI derivative and phasing was achieved with remote SAD methods, using a 1.77- $\AA$ 

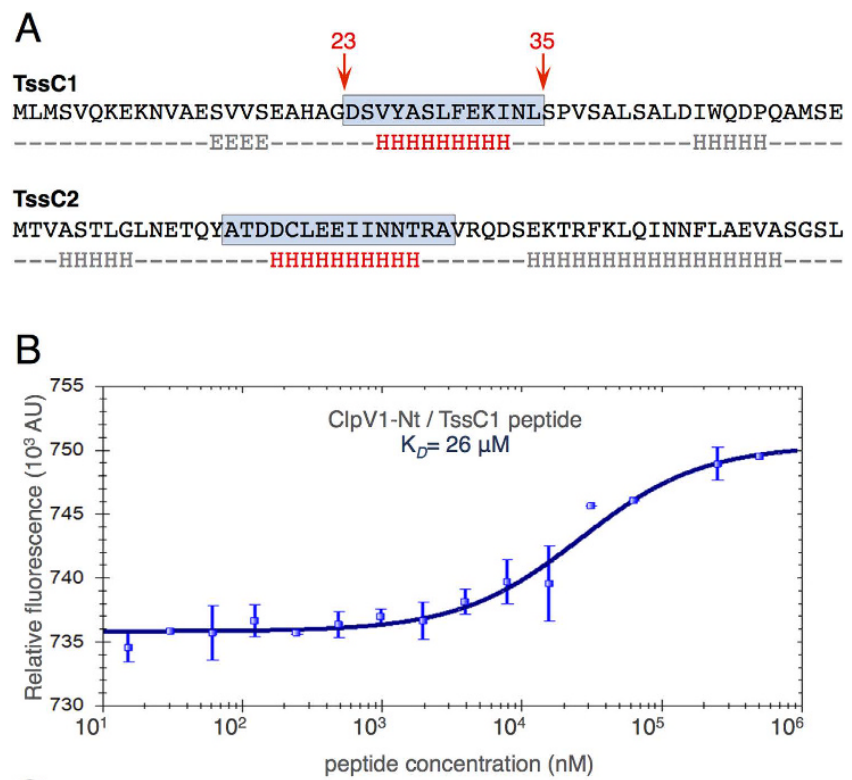

C

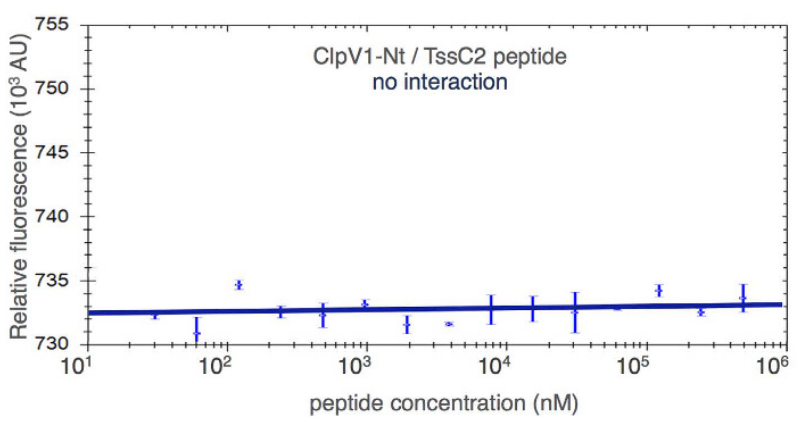

Figure 3. The TssC1 N-terminal helix specifically binds to the ClpV1 N-terminal domain. (A) Sequences of the $\mathrm{Tss} C 1$ and $\mathrm{Tss} C 2 \mathrm{~N}$-terminal regions. The secondary structure predictions (using JPRED ${ }^{64}$ ) are indicated under the sequence, and the TssC1 and TssC2 N-terminal helices (corresponding to the peptides used in this study) are highlighted by the blue frames. (B,C) Microscale thermophoresis interaction of the TssC1 (B) or TssC2 (C) N-terminal helical peptide with ClpV1-Nt. The MST signal change (expressed as relative fluorescence) that reflects titration of the unlabeled Tss C peptide (in nM) to a constant amount of fluorescently labeled ClpV1-Nt domain was measured. Error bars represent the results from three independent experiments.

wavelength for data collection. The amino-acid chain could be traced between residues 1 and 163 . The structure comprises $10 \alpha$-helices (H0 to H9) (Fig. 4), with overall dimensions of a flat disc of $50 \times 46 \times 28 \AA$. Interestingly, helix $\mathrm{H} 0$ is oriented perpendicular to helix $\mathrm{H} 1$, delimiting a groove.

Crystals of ClpV1-Nt in complex with the TssC1 peptide were obtained. The crystals belong to space group $\mathrm{P} 22_{1} 2_{1}$ and diffracted to 2.5- $\AA$ resolution (Supplemental Table S2). The structure of the complex was determined by molecular replacement using the uncomplexed structure (Fig. 5A,B). ClpV1-Nt is defined between residues 6 and 162 in the electron density map and its structure shows that it is not subjected to significant variation compared to the unbound ClpV1-Nt domain (root mean square deviation of $0.8 \AA$ ). The TssC1 N-terminal peptide is well defined in the electron density map between the residue Asp23 and Leu35. The peptide forms a 2-turn $\alpha$-helix between Ser24 and Phe30 (SVYASLF), while the rest of the peptide (EKINL) is elongated (Fig. 5A,B). PISA analysis shows that the interaction between ClpV1-Nt and the TssC1 peptide covers $622 \AA^{2}$ on the peptide ( $38 \%$ of the total surface) and $520 \AA^{2}$ on the ClpV1 N-terminal domain (6.6\% of the total surface) (Fig. 5C, Supplemental Table S3). The TssC1 peptide accommodates in the ClpV1-Nt groove delimited by helices $\mathrm{H} 0$ and $\mathrm{H} 1$, and the H4-H5 loop (Figs 4A and 5A-E, Supplemental Table S3). Most residues of the peptide are involved in the interaction, with the exception of Ser24 and Ala27. The most significant interactions involve Tyr26, Leu29, Phe30, Lys32, Ile33 and Asn34, the three last residues belonging to the elongated stretch (Fig. 5D,E, Supplemental Table S3). The co-structure of ClpV1-Nt with the N-terminal peptide of TssC1 therefore reveals a mode of binding distinct from previous co-structures ${ }^{43}$ (see Discussion).

Mutagenesis studies confirm the ClpV1-TssC1 interface shown in the co-crystal. To test the physiological relevance of the ClpV1-Nt/TssC1 peptide co-structure, we engineered point mutations within the $\mathrm{ClpV} 1$ groove and the TssC1 N-terminal region, and tested their effects on ClpV1-TssC1 interaction and T6SS function. The Glu24-to-Lys (ClpV1 ${ }^{\mathrm{EK}}$ mutant) and Arg87-to-Glu (ClpV1 ${ }^{\mathrm{RE}}$ mutant) substitutions (as well as the 
A
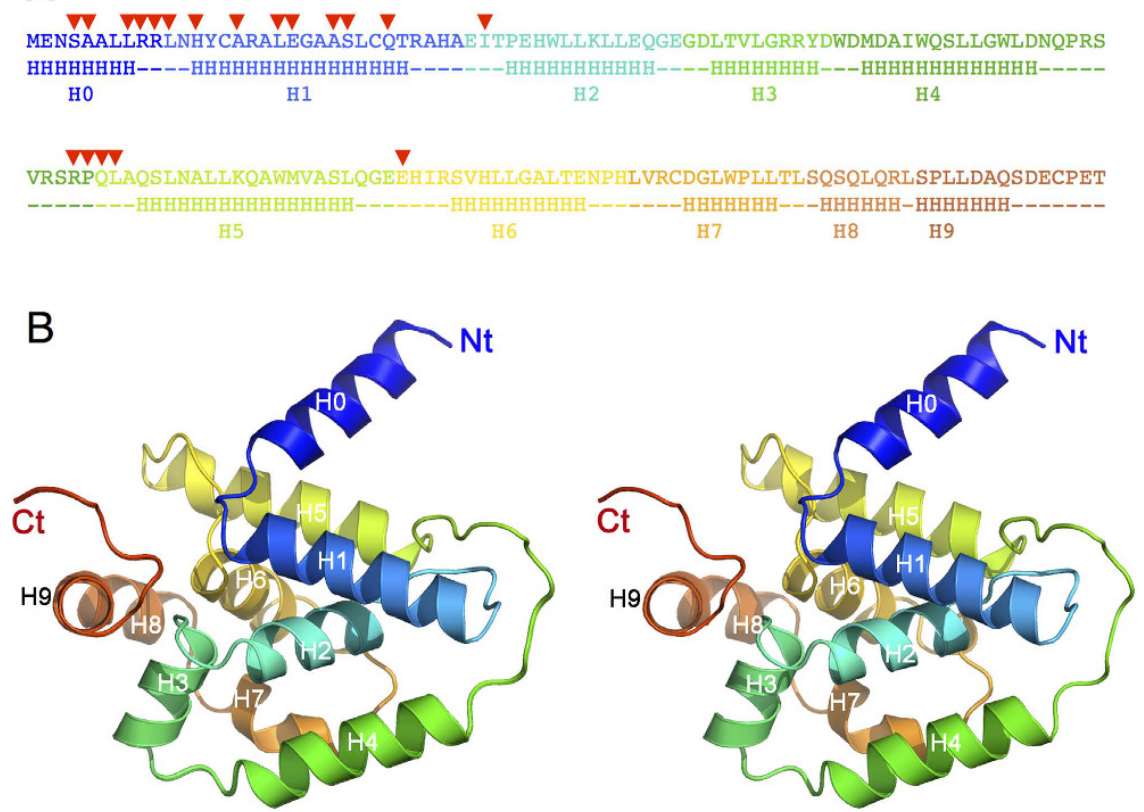

Figure 4. Crystal structure of the ClpV1 N-terminal domain. (A) Rainbow colored sequence of the ClpV1 $\mathrm{N}$-terminal domain. The secondary structures are indicated below the sequence. The residues involved in the interaction with the TssC1 peptide (see Fig. 5) are indicated by the red arrowheads. (B) Stereoview of the ClpV1 $\mathrm{N}$-terminal domain. The structure is represented as a rainbow colored ribbon. Helices are numbered $\mathrm{H} 0-\mathrm{H} 8$.

double mutant variant, $\mathrm{ClpV} 1^{\mathrm{EKRE}}$ ) were introduced in $\mathrm{ClpV} 1$. These mutations did not interfere with ClpV1 oligomerization (Fig. 6A). The Glu31-to-Lys and Lys32-to-Glu substitutions were introduced in TssC1 (TssC1 ${ }^{\mathrm{EKKE}}$ mutant). Here again, these mutations did not prevent the ability of TssC1 to oligomerize or to interact with TssB1 (Fig. 6A), two complexes previously described in $\mathrm{EAEC}^{60}$. However, all these substitutions affected partly or totally ClpV1-TssC1 complex formation (Fig. 6A). While the ClpV1 RE mutation had only a mild effect on the interaction, the ClpV1 EK and EKRE and the TssC1 EKKE mutations prevented formation of the ClpV1-TssC1 complex. The ClpV1 and TssC1 variants were then tested for their ability to support T6SS function. The two double mutants, $\mathrm{ClpV} 1^{\mathrm{EKRE}}$ and TssC1 ${ }^{\mathrm{EKKE}}$, did not restore the anti-bacterial activity of $\Delta c l p V 1$ (Fig. $6 \mathrm{~B}$ ) and $\Delta t s s C 1$ (Fig. 6C) EAEC cells, respectively. By contrast the ClpV1 RE and EK single substitutions caused a 9- and 310-fold decrease of the function of the EAEC Sci-1 T6SS, respectively (Fig. 6B).

\section{Discussion}

Bacterial genomes may encode several copies of Type VI secretion gene clusters. Although these gene clusters are usually subjected to different regulatory controls and expressed in different conditions, distinct Type VI secretion machineries may co-exist in the same bacterial cell ${ }^{1,2}$. There is therefore a need to control proper assembly of these machines and to allow recruitment of cognate subunits. Indeed, previous studies have demonstrated that specificity operates during machine assembly, more specifically for the interaction between the inner tube Hcp subunit and its cognate TssB sheath protein in enteroaggregative E. coli ${ }^{21}$. Here, we show that a second level of specificity exists, at the stage of sheath disassembly/recycling. The EAEC 17-2 strain genome encodes two T6SS gene clusters $^{44,58}$, each containing its own ClpV ATPase. These two proteins share 38/65\% sequence identity/similarity (Supplemental Figure S1). Here, we show that these two proteins are not interchangeable and that ClpV2 cannot rescue the anti-bacterial defects of a $\Delta c l p V 1$ strain, and vice versa. Using a combination of bacterial two-hybrid, co-immunoprecipitation and in vitro microscale thermophoresis assays, we provide evidence that specificity is dictated by binding of the Tss $\mathrm{C} \mathrm{N}$-terminal helix to the ClpV N-terminal domain. The $\mathrm{N}$-terminal regions of TssC1 and TssC2 (residues 1-163) are much more divergent (20/53\% identity/similarity) than full-length proteins $(38 / 65 \%)$ (Supplemental Figure S1). The crystal structure of the ClpV1 N-terminal domain in complex with the TssC1 N-terminal helix further showed that the TssC1 helix inserts into a cleft delimitated by the $\mathrm{H} 0$ and $\mathrm{H} 1$ helices and the H4-H5 loop (Figs 4A, 5 and 6, Supplemental Table S3). Interestingly, binding of the TssC1 N-terminal helix to ClpV1-Nt does not cause a significant conformational change within ClpV. This situation is similar to that reported for the $V$. cholerae $\mathrm{ClpV}_{\mathrm{Vc}_{\mathrm{c}}}$-VipB complex (see below) $)^{41}$. The weak $\mathrm{K}_{D}$ value $(26 \mu \mathrm{M}$ ) is also very similar to that measured in $V$. cholerae $(39 \mu \mathrm{M})^{41}$. However, the $\mathrm{K}_{D}$ values obtained in vitro might not represent the in vivo situation. In vivo, it is assumed that the ClpV ATPase is recruited to the contracted sheath ${ }^{15,18,22,61}$, and therefore hundreds of TssC N-terminal helices are available, probably resulting in avidity higher by several orders of magnitudes as compared to the affinity of isolated fragments.

The structure of the T6SS contracted sheath ${ }^{23,24,62}$ revealed that the TssC N-terminal helices are not visible in the electron density map, probably due to their high flexibility. However, the position of the first visible Tss C residue (residue 61, pointed by the red arrow in Fig. 7A) strongly suggests that the N-terminal VipB/TssC helix 
A

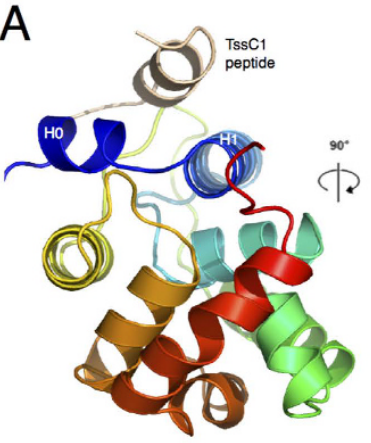

D

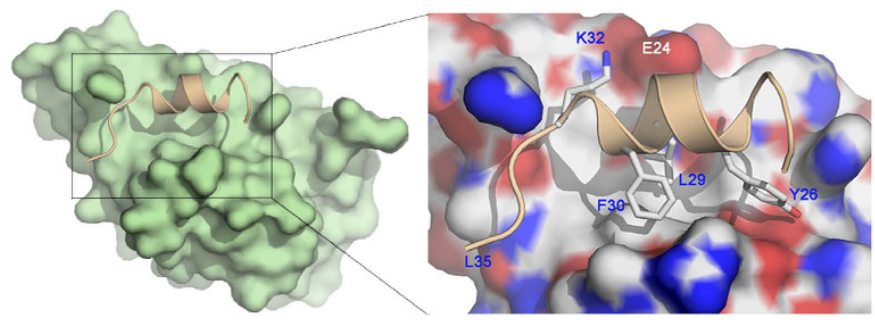

C
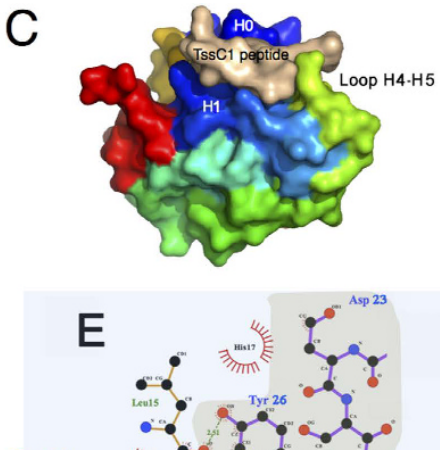

E

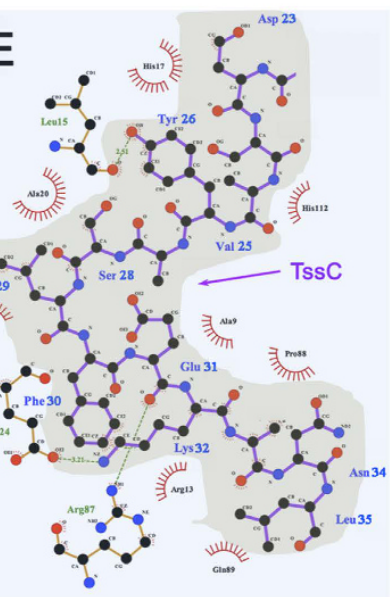

Figure 5. Crystal structure of the ClpV1 N-terminal domain complexed to the TssC1 N-terminal helix. $(\mathbf{A}, \mathbf{B})$ Ribbon view of the ClpV1-Nt/TssC1 peptide complex. The ClpV1 N-terminal domain is rainbow colored and the TssC1 peptide is shown in beige. The view in (B) is rotated by $90^{\circ}$ compared to (A) and the uncomplexed ClpV1 N-terminal domain is superimposed and colored grey. (C) Surface representation of the complex. The ClpV1 N-terminal domain is rainbow colored and the TssC1 peptide is colored beige. (D) Surface view of the ClpV1 N-terminal domain (green) in complex with the TssC1 peptide represented as a ribbon (beige). The inset shows a close-up of the binding crevice, with the surface colored according to electrostatics (blue/red, positively/negatively charged residues; white, hydrophobic residues). (E) Detailed ball-and-stick scheme of the interaction. The side chains of the residues at the ClpV1-TssC1 interface of the ClpV1 N-terminal domain (blue area) are represented with brown bonds whereas that of the $\mathrm{Tss} C 1$ peptide (grey area) are represented with violet bonds. Tss $\mathrm{C} 1$ residues are indicated in blue whereas the side chains of ClpV1 residues involved in the interaction are indicated in green (scheme made with Ligplot $+{ }^{65}$ ).

protrudes far out of the sheath cylinder and hence might be accessible to ClpV. This observation supports the current model in which sheath contraction extricates the TssC N-terminal helix, leading to ClpV recruitment and sheath subunits recycling ${ }^{18,22}$. However, this model assumes that the Tss $\mathrm{C} \mathrm{N}$-terminal helix is buried within the extended sheath. To date, the molecular structure of the T6SS sheath under the extended conformation is not available. In a recent study, a low resolution model of the extended V. cholerae VipAB sheath was modeled using the low resolution EM map of the extended T4 phage tail sheath ${ }^{23}$. By superimposing the VipAB EM map to the gp18 T4 phage sheath protein, gross features of the sheath structure were obtained ${ }^{23}$. To obtain high-resolution details, we applied this approach by fitting the recently released VipAB molecular model into the extended T4 phage tail sheath. In the extended model, VipB residue 61 is also located near the sheath surface, but packed against the cylinder surface (red arrows in Fig. 7B). We noticed an empty cavity nearby residue 61 that is large enough to accommodate the VipB N-terminal segment 1-60 (Fig. 7B). In this configuration, the TssC N-terminal helix will be buried and therefore not accessible to ClpV.

The structures of the $V$. cholerae (PDB 3ZRI) ${ }^{41}$ and EAEC (this study) ClpV N-terminal domains share very close folds (Fig. 8). They display an elongated crevice at the same location, in which the TssC (VipB) helix inserts. While the overall scheme is the same, one can notice several differences. First, the position and orientation of the $V$. cholerae VipB and EAEC TssC1 N-terminal $\alpha$-helices within the groove are slightly different. The TssC1 peptide starts with an alpha helical structure followed by an extended segment (Fig. 8). By contrast, the VipB peptide is entirely helical and locates within $\mathrm{ClpV}_{\mathrm{Vc}}$ at a position that is occupied by the TssC1 extended segment in ClpV1 (Fig. 8). This indicates, as already suggested ${ }^{43}$, that although ClpV exhibits a common fold in different T6SS, the nature and position of residues involved in Tss C binding vary significantly enough to elicit variations in the mode of interaction. In addition, the ClpV1 groove that accommodates the Tss $\mathrm{C} 1$ helix is constituted of a hydrophobic central area borded by charged residues, Glu24 of $\mathrm{H} 1$ in contact with the Lys 29 residue of TssC1, and Arg87 of the $\mathrm{H} 4-\mathrm{H} 5$ loop in contact with a main-chain carboxylic group of TssC1 (Fig. 5D,E). It has been proposed that ClpV proteins could be categorized in two phylogenetic groups differing in the nature of residues within the groove (charged or uncharged residues) ${ }^{43}$. It has also been suggested that $\mathrm{ClpV}$ proteins with charged grooves cannot bind TssC N-terminal helices and require the assistance of an accessory protein, Tag ${ }^{43,63}$. However, although the EAEC ClpV1 protein presents a central hydrophobic groove borded by charged residues, (i) it is able to bind directly to the TssC N-terminal helix, (ii) the ClpV1 charged residues are critical for the ClpV1-TssC1 interaction, 
A

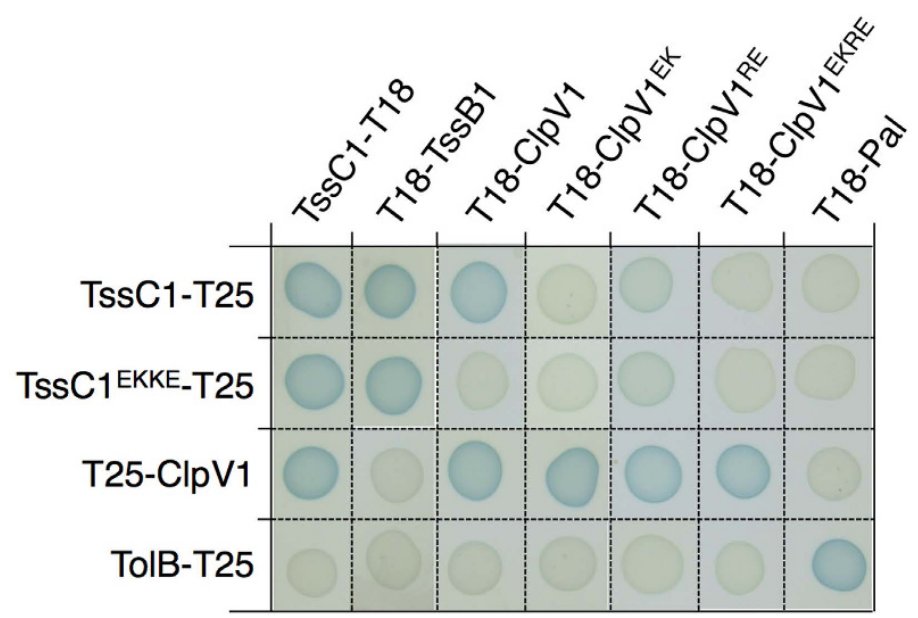

B
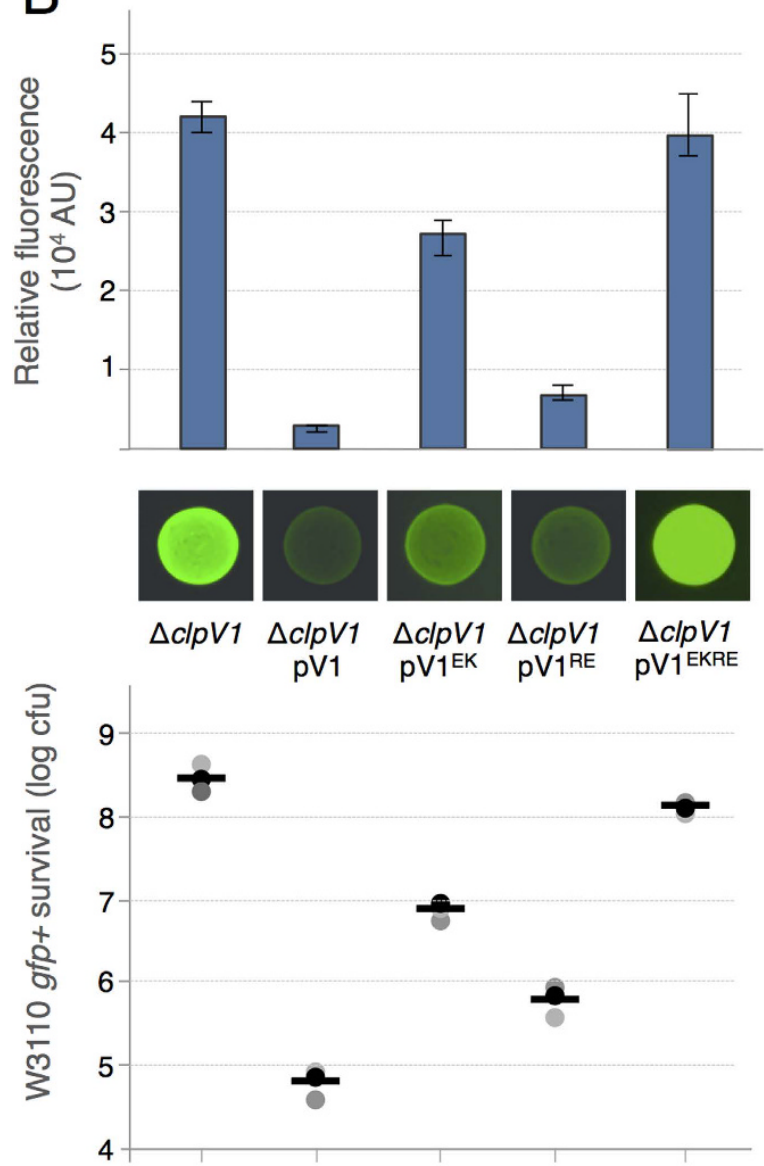

C
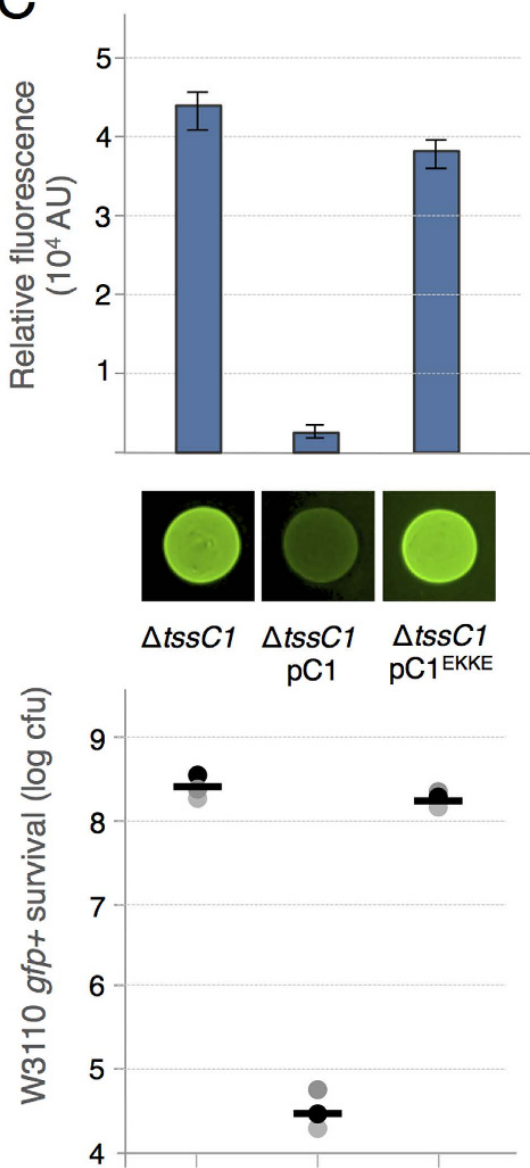

Figure 6. Charged residues within the $\mathrm{ClpV} 1$ groove and the TssC1 peptide mediate the ClpV1-TssC1 interaction. (A) Bacterial two-hybrid assay. BTH101 reporter cells producing the indicated wild-type or mutated proteins fused to the T18 or T25 domains of the Bordetella adenylate cyclase were spotted on X-Gal indicator plates. The blue color of the colony reflects the interaction between the two proteins. (B,C) Sci1-dependent antibacterial growth inhibition. Prey cells $\left(\mathrm{W} 3110 \mathrm{gfp} \mathrm{p}^{+}, \mathrm{kan}^{\mathrm{R}}\right)$ were mixed with the indicated attacker cells, spotted onto sci-1-inducing medium (SIM) agar plates and incubated for 4 hours at $37^{\circ} \mathrm{C}$. The image of a representative bacterial spot is shown and the relative fluorescent levels (in arbitrary units, AU) are indicated in the upper graph. The number of recovered E. coli prey cells is indicated in the lower graph (in $\log _{10}$ of colony-forming units ( $\mathrm{cfu})$ ). The circles indicate values from three independent assays, and the average is indicated by the bar. 

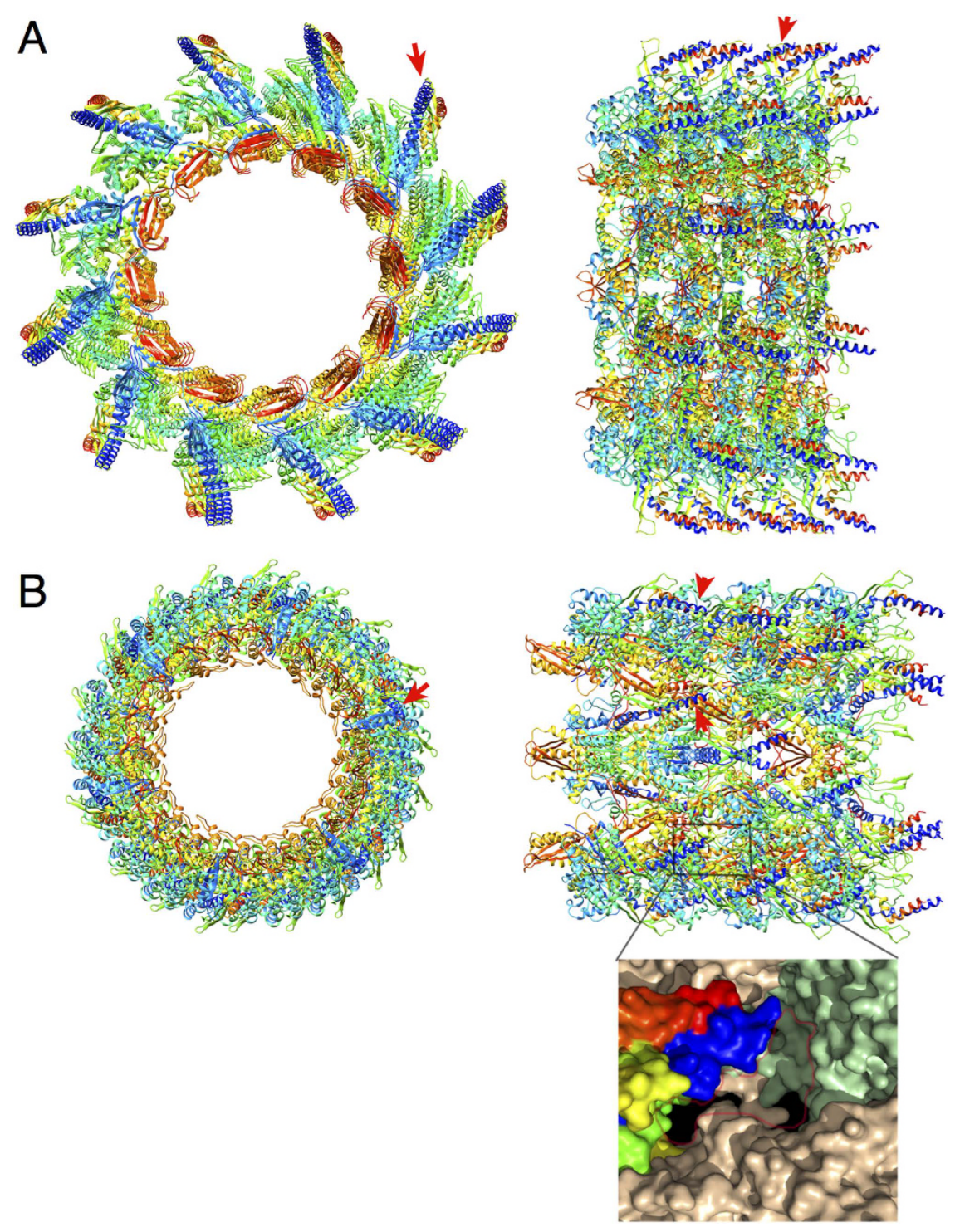

Figure 7. Position of TssC N-terminal helix in the contracted and extended T6SS sheath models.

(A) Contracted V. cholerae VipAB sheath atomic structure ${ }^{24}$ (right, top view; left, side view). Four sheath rows are shown (right, top view; left, side view). The red arrows indicate the VipB (TssC) first visible residue.

(B) Extended V. cholerae VipAB sheath molecular model. The inset shows a close-up of the VipB N-terminus and the flanking cavity (surligned in red) that could accommodate the VipB 1-60 fragment.



Figure 8. Structural comparison between the EAEC and Vibrio cholerae ClpV N-terminal domains in

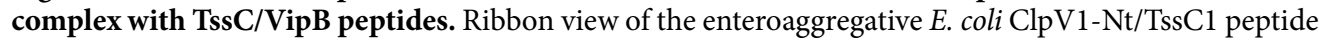
(ClpV in blue, peptide in orange; $\mathrm{PDB} 4 \mathrm{HH} 6)$ superimposed to the V. cholerae ClpV-Nt/VipB peptide (ClpV in green, peptide in pink; PDB $3 Z R J^{41}$ ). 
and (ii) no TagJ homologue is encoded within the EAEC genome. These observations suggest that interaction between ClpV1 and TssC1 in EAEC represents a third mode of binding dependent on a partially charged interface but independent on TagJ. This variability in the mechanism of ClpV binding to TssC supports the notion that specific determinants regulate recruitment of ClpV to contracted sheaths in cells with distinct T6SS machines.

\section{References}

1. Bingle, L. E., Bailey, C. M. \& Pallen, M. J. Type VI secretion: a beginner's guide. Curr Opin Microbiol 11, 3-8, doi: S13695274(08)00007-6 (2008).

2. Cascales, E. The type VI secretion toolkit. EMBO Rep 9, 735-741, doi: 10.1038/embor.2008.131 (2008).

3. Boyer, F., Fichant, G., Berthod, J., Vandenbrouck, Y. \& Attree, I. Dissecting the bacterial type VI secretion system by a genome wide in silico analysis: what can be learned from available microbial genomic resources? BMC Genomics 10, 104, doi: 10.1186/1471-216410-104 (2009).

4. Coyne, M. J., Roelofs, K. G. \& Comstock, L. E. Type VI secretion systems of human gut Bacteroidales segregate into three genetic architectures, two of which are contained on mobile genetic elements. BMC Genomics 17, 58, doi: 10.1186/s12864-016-2377-z (2016).

5. Hood, R. D. et al. A type VI secretion system of Pseudomonas aeruginosa targets a toxin to bacteria. Cell Host Microbe 7, 25-37, doi: 10.1016/j.chom.2009.12.007 (2010).

6. Russell, A. B. et al. Type VI secretion delivers bacteriolytic effectors to target cells. Nature 475, 343-347, doi: 10.1038/nature10244 (2011).

7. Durand, E., Cambillau, C., Cascales, E. \& Journet, L. VgrG, Tae, Tle, and beyond: the versatile arsenal of Type VI secretion effectors. Trends Microbiol 22, 498-507, doi: 10.1016/j.tim.2014.06.004 (2014).

8. Alcoforado Diniz, J., Liu, Y. C. \& Coulthurst, S. J. Molecular weaponry: diverse effectors delivered by the Type VI secretion system. Cell Microbiol 17, 1742-1751, doi: 10.1111/cmi.12532 (2015).

9. Sana, T. G. et al. Salmonella Typhimurium utilizes a T6SS-mediated antibacterial weapon to establish in the host gut. Proc Natl Acad Sci USA 113, 5044-5051, doi: 10.1073/pnas.16088581132016 (2016).

10. Pukatzki, S., Ma, A. T., Revel, A. T., Sturtevant, D. \& Mekalanos, J. J. Type VI secretion system translocates a phage tail spike-like protein into target cells where it cross-links actin. Proc Natl Acad Sci USA 104, 15508-15513, doi: 10.1073/pnas.0706532104 (2007).

11. Pukatzki, S. et al. Identification of a conserved bacterial protein secretion system in Vibrio cholerae using the Dictyostelium host model system. Proc Natl Acad Sci USA 103, 1528-1533, doi: 10.1073/pnas.0510322103 (2006).

12. Durand, E. et al. Crystal structure of the VgrG1 actin cross-linking domain of the Vibrio cholerae type VI secretion system. J Biol Chem 287, 38190-38199, doi: 10.1074/jbc.M112.390153 (2012).

13. Sana, T. G. et al. Internalization of Pseudomonas aeruginosa strain PAO1 into epithelial cells is promoted by interaction of a T6SS effector with the microtubule network. MBio 6, e00712, doi: 10.1128/mBio.00712-15 (2015).

14. Cascales, E. \& Cambillau, C. Structural biology of type VI secretion systems. Philos Trans R Soc Lond B Biol Sci 367, 1102-1111, doi: $10.1098 /$ rstb.2011.0209 (2012).

15. Zoued, A. et al. Architecture and assembly of the Type VI secretion system. Biochim Biophys Acta 1843, 1664-1673, doi: 10.1016/j. bbamcr.2014.03.018 (2014).

16. Basler, M. Type VI secretion system: secretion by a contractile nanomachine. Philos Trans R Soc Lond B Biol Sci 370, doi: 10.1098/ rstb.2015.0021 (2015).

17. Cianfanelli, F. R., Monlezun, L. \& Coulthurst, S. J. Aim, load, fire: the type VI secretion system, a bacterial nanoweapon. Trends Microbiol 24, 51-62, doi: 10.1016/j.tim.2015.10.005 (2016).

18. Bonemann, G., Pietrosiuk, A. \& Mogk, A. Tubules and donuts: a type VI secretion story. Mol Microbiol 76, 815-821, doi: 10.1111/j.1365-2958.2010.07171.x (2010).

19. Leiman, P. G. \& Shneider, M. M. Contractile tail machines of bacteriophages. Adv Exp Med Biol 726, 93-114, doi: 10.1007/978-14614-0980-9_5(2012).

20. Basler, M., Pilhofer, M., Henderson, G. P., Jensen, G. J. \& Mekalanos, J. J. Type VI secretion requires a dynamic contractile phage tail-like structure. Nature 483, 182-186, doi: 10.1038/nature10846 (2012).

21. Brunet, Y. R., Henin, J., Celia, H. \& Cascales, E. Type VI secretion and bacteriophage tail tubes share a common assembly pathway. EMBO Rep, doi: 10.1002/embr.201337936 (2014).

22. Bonemann, G., Pietrosiuk, A., Diemand, A., Zentgraf, H. \& Mogk, A. Remodelling of VipA/VipB tubules by ClpV-mediated threading is crucial for type VI protein secretion. EMBO J 28, 315-325, doi: 10.1038/emboj.2008.269 (2009).

23. Kube, S. et al. Structure of the VipA/B type VI secretion complex suggests a contraction-state-specific recycling mechanism. Cell Rep 8, 20-30, doi: 10.1016/j.celrep.2014.05.034 (2014).

24. Kudryashev, M. et al. Structure of the type VI secretion system contractile sheath. Cell 160, 952-962, doi: 10.1016/j.cell.2015.01.037 (2015).

25. Leiman, P. G. et al. Type VI secretion apparatus and phage tail-associated protein complexes share a common evolutionary origin. Proc Natl Acad Sci USA 106, 4154-4159, doi: 10.1073/pnas.0813360106 (2009).

26. Shneider, M. M. et al. PAAR-repeat proteins sharpen and diversify the type VI secretion system spike. Nature 500, 350-353, doi: 10.1038 /nature12453 (2013).

27. Alcoforado Diniz, J. \& Coulthurst, S. J. Intraspecies competition in Serratia marcescens is mediated by Type VI-secreted Rhs effectors and a conserved effector-associated accessory protein. J Bacteriol 197, 2350-2360, doi: 10.1128/JB.00199-15 (2015).

28. Unterweger, D. et al. Chimeric adaptor proteins translocate diverse type VI secretion system effectors in Vibrio cholerae. EMBO J 34, 2198-2210, doi: 10.15252/embj.201591163 (2015).

29. Flaugnatti, N. et al. A phospholipase A1 antibacterial Type VI secretion effector interacts directly with the C-terminal domain of the VgrG spike protein for delivery. Mol Microbiol 99, 1099-1118, doi: 10.1111/mmi.13292 (2016).

30. English, G., Byron, O., Cianfanelli, F. R., Prescott, A. R. \& Coulthurst, S. J. Biochemical analysis of TssK, a core component of the bacterial Type VI secretion system, reveals distinct oligomeric states of TssK and identifies a TssK-TssFG sub-complex. Biochem J, doi: 10.1042/BJ20131426(2014).

31. Brunet, Y. R., Zoued, A., Boyer, F., Douzi, B. \& Cascales, E. The type VI secretion TssEFGK-VgrG phage-like baseplate is recruited to the TssJLM membrane complex via multiple contacts and serves as assembly platform for tail tube/sheath polymerization. PLoS Genet 11, e1005545, doi: 10.1371/journal.pgen.1005545 (2015).

32. Gerc, A. J. et al. Visualization of the Serratia type VI secretion system reveals unprovoked attacks and dynamic assembly. Cell Rep 12, 2131-2142, doi: 10.1016/j.celrep.2015.08.053 (2015).

33. Zoued, A. et al. TssK is a trimeric cytoplasmic protein interacting with components of both phage-like and membrane anchoring complexes of the Type VI secretion system. J Biol Chem 288, 27031-27041, doi: 10.1074/jbc.M113.499772 (2013).

34. Aschtgen, M. S., Bernard, C. S., De Bentzmann, S., Lloubes, R. \& Cascales, E. SciN is an outer membrane lipoprotein required for type VI secretion in enteroaggregative Escherichia coli. J Bacteriol 190, 7523-7531, doi: 10.1128/JB.00945-08 (2008).

35. Aschtgen, M. S., Gavioli, M., Dessen, A., Lloubes, R. \& Cascales, E. The SciZ protein anchors the enteroaggregative Escherichia coli Type VI secretion system to the cell wall. Mol Microbiol, doi: 10.1111/j.1365-2958.2010.07028.x (2010). 
36. Felisberto-Rodrigues, C. et al. Towards a structural comprehension of bacterial type VI secretion systems: characterization of the TssJ-TssM complex of an Escherichia coli pathovar. PLoS Pathog 7, e1002386, doi: 10.1371/journal.ppat.1002386 (2011).

37. Aschtgen, M. S., Zoued, A., Lloubes, R., Journet, L. \& Cascales, E. The C-tail anchored TssL subunit, an essential protein of the enteroaggregative Escherichia coli Sci-1 Type VI secretion system, is inserted by YidC. Microbiologyopen 1, 71-82, doi: 10.1002/ mbo3.9 (2012).

38. Durand, E. et al. Structural characterization and oligomerization of the TssL protein, a component shared by bacterial type VI and type IVb secretion systems. J Biol Chem 287, 14157-14168, doi: 10.1074/jbc.M111.338731 (2012).

39. Durand, E. et al. Biogenesis and structure of a type VI secretion membrane core complex. Nature 523, 555-560, doi: 10.1038/ nature14667 (2015)

40. Brunet, Y. R., Espinosa, L., Harchouni, S., Mignot, T. \& Cascales, E. Imaging type VI secretion-mediated bacterial killing. Cell Rep 3, 36-41, doi: 10.1016/j.celrep.2012.11.027 (2013).

41. Pietrosiuk, A. et al. Molecular basis for the unique role of the AAA + chaperone ClpV in type VI protein secretion. J Biol Chem 286, 30010-30021, doi: 10.1074/jbc.M111.253377 (2011).

42. Basler, M. \& Mekalanos, J. J. Type 6 secretion dynamics within and between bacterial cells. Science 337, 815, doi: 10.1126/ science.1222901 (2012).

43. Forster, A. et al. Coevolution of the ATPase ClpV, the sheath proteins TssB and TssC, and the accessory protein TagJ/HsiE1 distinguishes type VI secretion classes. J Biol Chem 289, 33032-33043, doi: 10.1074/jbc.M114.600510 (2014).

44. Journet, L. \& Cascales, E. The Type VI secretion system in Escherichia coli and related species. EcoSal Plus 7, doi: 10.1128/ecosalplus. ESP-0009-2015 (2016).

45. Bernard, C. S., Brunet, Y. R., Gueguen, E. \& Cascales, E. Nooks and crannies in type VI secretion regulation. J Bacteriol 192, 3850-3860, doi: 10.1128/JB.00370-10 (2010)

46. Zaslaver, A. et al. A comprehensive library of fluorescent transcriptional reporters for Escherichia coli. Nat Methods 3, 623-628, doi: 10.1038/nmeth895 (2006).

47. van den Ent, F. \& Lowe, J. RF cloning: a restriction-free method for inserting target genes into plasmids. J Biochem Biophys Methods 67, 67-74, doi: 10.1016/j.jbbm.2005.12.008 (2006).

48. Karimova, G., Pidoux, J., Ullmann, A. \& Ladant, D. A bacterial two-hybrid system based on a reconstituted signal transduction pathway. Proc Natl Acad Sci USA 95, 5752-5756 (1998).

49. Battesti, A. \& Bouveret, E. The bacterial two-hybrid system based on adenylate cyclase reconstitution in Escherichia coli. Methods 58, 325-334, doi: 10.1016/j.ymeth.2012.07.018 (2012)

50. Kabsch, W. Xds. Acta Crystallogr D Biol Crystallogr 66, 125-132, doi: S0907444909047337 (2010).

51. Schneider, T. R. \& Sheldrick, G. M. Substructure solution with SHELXD. Acta Crystallogr D Biol Crystallogr 58, 1772-1779 (2002).

52. McCoy, A. J. et al. Phaser crystallographic software. J Appl Crystallogr 40, 658-674, doi: 10.1107/S0021889807021206 (2007).

53. Cowtan, K. Recent developments in classical density modification. Acta Crystallogr D Biol Crystallogr 66, 470-478, doi: 10.1107/ S090744490903947X (2010).

54. Cowtan, K. The Buccaneer software for automated model building. 1. Tracing protein chains. Acta Crystallogr D Biol Crystallogr 62, 1002-1011, doi: 10.1107/S0907444906022116 (2006).

55. Emsley, P. \& Cowtan, K. Coot: model-building tools for molecular graphics. Acta Crystallogr D Biol Crystallogr 60, 2126-2132 (2004).

56. Blanc, E. et al. Refinement of severely incomplete structures with maximum likelihood in BUSTER-TNT. Acta Crystallogr D Biol Crystallogr 60, 2210-2221, doi: S09074444904016427 (2004).

57. Brunet, Y. R., Bernard, C. S., Gavioli, M., Lloubes, R. \& Cascales, E. An epigenetic switch involving overlapping fur and DNA methylation optimizes expression of a type VI secretion gene cluster. PLoS Genet 7, e1002205, doi: 10.1371/journal.pgen.1002205 (2011).

58. Dudley, E. G., Thomson, N. R., Parkhill, J., Morin, N. P. \& Nataro, J. P. Proteomic and microarray characterization of the AggR regulon identifies a pheU pathogenicity island in enteroaggregative Escherichia coli. Mol Microbiol 61, 1267-1282 (2006).

59. Kress, W., Maglica, Z. \& Weber-Ban, E. Clp chaperone-proteases: structure and function. Res Microbiol 160, 618-628, doi: 10.1016/j. resmic.2009.08.006 (2009).

60. Zhang, X.Y., Brunet, Y.R., Logger, L., Douzi, B., Cambillau, C., Journet, L. \& Cascales, E. Dissection of the TssB-TssC interface during type VI secretion sheath complex formation. PLoS One 8, e81074. doi: 10.1371/journal.pone.0081074 (2013).

61. Kapitein, N. et al. ClpV recycles VipA/VipB tubules and prevents non-productive tubule formation to ensure efficient type VI protein secretion. Mol Microbiol 87, 1013-1028, doi: 10.1111/mmi.12147 (2013).

62. Clemens, D. L., Ge, P., Lee, B. Y., Horwitz, M. A. \& Zhou, Z. H. Atomic structure of T6SS reveals interlaced array essential to function. Cell 160, 940-951, doi: 10.1016/j.cell.2015.02.005 (2015).

63. Lossi, N. S. et al. The archetype Pseudomonas aeruginosa proteins TssB and TagJ form a novel subcomplex in the bacterial type VI secretion system. Mol Microbiol 86, 437-456, doi: 10.1111/j.1365-2958.2012.08204.x (2012).

64. Cole, C., Barber, J. D. \& Barton, G. J. The Jpred 3 secondary structure prediction server. Nucleic Acids Res 36, W197-201, doi: gkn238 (2008).

65. Laskowski, R. A. \& Swindells, M. B. LigPlot+: multiple ligand-protein interaction diagrams for drug discovery. J Chem Inf Model 51, 2778-2786, doi: $10.1021 / \mathrm{ci} 200227 \mathrm{u}(2011)$

\section{Acknowledgements}

We thank the members of the Cascales and Cambillau research groups for insightful discussions and the Soleil synchrotron (Saint-Aubin, France) for beamline allocation. This work was supported by the Centre Nationale de la Recherche Scientifique (CNRS), the Aix-Marseille Université (AMU), Agence Nationale de la Recherche (ANR) and Fondation pour la Recherche Médicale (FRM) research grants (ANR-10-JCJC-1303-03, ANR14-CE14-0006-02, DEQ2011-0421282). Work of Valentine Lensi was performed in fulfillment of an Aix-Marseille Univ BIO99 project. Anant Kumar was supported by a Charpak Research Excellence scholarship from the Agence Française pour la promotion de l'enseignement supérieur, l'accueil et la mobilité internationale (Campus France).

\section{Author Contributions}

B.D., S.S. and S.B. purified the proteins and performed the microscale thermophoresis experiments. B.D., S.S., P.L. and C.C. solved the X-ray structures. Y.R.B., V.L. and E.C. performed the co-immunoprecipitations and anti-bacterial activity assays. A.S. and L.J. performed the bacterial two-hybrid assays. E.C. and C.C. supervised the experiments. B.D., E.C. and C.C. wrote the manuscript. Every author reviewed the manuscript prior to submission. 


\section{Additional Information}

Supplementary information accompanies this paper at http://www.nature.com/srep

Competing financial interests: The authors declare no competing financial interests.

How to cite this article: Douzi, B. et al. Structure and specificity of the Type VI secretion system ClpV-TssC interaction in enteroaggregative Escherichia coli. Sci. Rep. 6, 34405; doi: 10.1038/srep34405 (2016).

(c) (i) This work is licensed under a Creative Commons Attribution 4.0 International License. The images or other third party material in this article are included in the article's Creative Commons license, unless indicated otherwise in the credit line; if the material is not included under the Creative Commons license, users will need to obtain permission from the license holder to reproduce the material. To view a copy of this license, visit http://creativecommons.org/licenses/by/4.0/

(C) The Author(s) 2016 